\title{
Loop Restricted Existential Rules and First-order Rewritability for Query Answering
}

\author{
Vernon Asuncion $^{a}$, Yan Zhang ${ }^{a, c}$, Heng Zhang ${ }^{b}$, Yun Bai ${ }^{a}$ and Weisheng Si ${ }^{a}$ \\ ${ }^{a}$ School of Computing Engineering and Mathematics \\ Western Sydney University, Australia \\ ${ }^{b}$ School of Computer Software, Tianjin University, China \\ ${ }^{c}$ Huazhong University of Science \& Technology, China
}

\begin{abstract}
In ontology-based data access (OBDA), the classical database is enhanced with an ontology in the form of logical assertions generating new intensional knowledge. A powerful form of such logical assertions is the tuple-generating dependencies (TGDs), also called existential rules, where Horn rules are extended by allowing existential quantifiers to appear in the rule heads. In this paper we introduce a new language called loop restricted (LR) TGDs (existential rules), which are TGDs with certain restrictions on the loops embedded in the underlying rule set. We study the complexity of this new language. We show that the conjunctive query answering (CQA) under the LR TGDs is decidable. In particular, we prove that this language satisfies the so-called bounded derivation-depth property (BDDP), which implies that the CQA is first-order rewritable, and its data complexity is in $\mathrm{AC}^{0}$. We also prove that the combined complexity of the CQA is EXPTIME complete, while the language membership is PSPACE complete. Then we extend the LR TGDs language to the generalised loop restricted (GLR) TGDs language, and prove that this class of TGDs still remains to be first-order rewritable and properly contains most of other first-order rewritable TGDs classes discovered in the literature so far.
\end{abstract}

\section{Introduction}

In ontology-based data access (OBDA), a database is enhanced with an ontology in the form of logical assertions generating new intensional knowledge, e.g., (Baader et al. 2016, Bienvenu 2016, Eiter, Lukasiewicz, and Predoiu 2016, Kontchakov, Rodriguez-Muro, and Zakharyaschev 2013; Nikolaou et al. 2017). A powerful form of such logical assertions is the tuple-generating dependencies (TGDs), also called existential rules. Generally speaking, TGDs are Horn rules extended by allowing the occurrence of existential quantification in the rule head. With this extension, it is able to reason about the existence of new or missing objects that are not represented in the underlying database (Baget et al. 2011, Patel-Schneider and Horrocks 2007).

Under the language of TGDs, queries are answered against an ontology represented by a set of TGDs and an

Copyright (c) 2018, Association for the Advancement of Artificial Intelligence (www.aaai.org). All rights reserved. input database. In particular, given a database instance $D$, a finite set $\Sigma$ of TGDs, and a query $q$, we want to decide whether $D \cup \Sigma \models q$. However, this problem is undecidable generally, due to the potential cyclic applications of TGDs in $\Sigma$ (Deutsch, Nash, and Remmel 2008).

In recent years, considerable research has been carried out to identify various expressive decidable classes of TGDs. So far several primary such classes have been discovered: weakly-acyclic class (Fagin et al. 2005); guarded class (Baget et al. 2011; Calì, Gottlob, and Kifer 2008; Calì, Gottlob, and Lukasiewicz 2012); sticky sets class (Calì, Gottlob, and Pieris 2012); and Shy programs class (Leone et al. 2012). By extending and combining these aforementioned classes, more decidable classes can be derived, such as glut-guardedness (weakacyclicity + guardedness) (Krötzsch and Rudolph 2011); weak-stickiness (weak-acyclicity + stickiness) (Calì, Gottlob, and Pieris 2012); model-faithful acyclicity (MFA) (Grau et al. 2013); and tameness (guardedness + stickiness) (Gottlob, Manna, and Pieris 2013).

Among all these decidable classes, some are of special interests for OBDA, i.e., the classes of first-order rewritable TGDs, where conjunctive query answering can be reduced to the evaluation of a first-order query over the database. As such, traditional database query techniques may be used for developing efficient query answering systems in OBDA, as demonstrated in Description Logics (Hansen et al. 2015 Kaminski, Nenov, and Grau 2014). So far, several useful first-order rewritable classes of TGDs have been discovered: acyclic TGDs, aGRD TGDs, linear and multilinear TGDs, sticky and sticky-join TGDs, while multi-linear and sticky-join TGDs generalise linear TGDs and sticky TGDs, respectively (Calì, Gottlob, and Lukasiewicz 2012 Calì, Gottlob, and Pieris 2012).

Civili and Rosati (Civili and Rosati 2012) further identified another first-order rewritable class called weakly recursive TGDs, and showed that by restricting to simple TGDs, weakly recursive class contains all other first-order rewritable classes.

Unfortunately, there are still real life scenarios that are simple and intuitive but not syntactically recognisable by any of the existing first-order rewritable TGDs classes, as illustrated by the following example.

Example 1. Consider a university research domain, where 
we have the following ontology $\Sigma_{\text {Research }}$ to represent its knowledge rules: We have the following ontology $\Sigma_{\text {Research }}$ to represent this domain:

$$
\begin{gathered}
\sigma_{1}: \operatorname{resAdvisor}(X, W) \rightarrow \operatorname{seniorStaff}(X), \\
\sigma_{2}: \text { seniorStaff }(X), \operatorname{advCommittee}(X, Y), \\
\operatorname{projDept}(X, Y) \rightarrow \exists W \operatorname{resAdvisor}(X, W) . \\
\sigma_{3}: \operatorname{resStudent}(W) \rightarrow \exists X Y \operatorname{resAdvisor}(X, W), \\
\quad \text { enrolDept }(W, Y), \operatorname{proj\operatorname {Dept}}(W, Y, Y), \\
\quad \operatorname{projDept}(X, Y, Y) .
\end{gathered}
$$

$\sigma_{1}$ says that if $X$ is a research advisor of someone, then $X$ must be a senior staff; $\sigma_{2}$ states that if $X$ is a senior staff and a member of the department $Y$ 's advisory committee, and $X$ also undertakes a project registered in department $Y$, then $X$ must be a research advisor of someonet; and $\sigma_{3}$ indicates that a research student $W$ must have an advisor and should undertake the project together with the advisor from the same department, while the project has to be also registered in the this department.

Through a careful examination, it is not difficult to see that $\Sigma_{\text {Research }}$ is not recognizable under the syntactic conditions of all currently known first-order rewritable TGDs classes. On the other hand, by unfolding the derivations on atoms seniorStaff $(X)$ and resAdvisor $(X, W)$ from $\Sigma_{\text {Research, }}$ it turns out that their derivations are always bounded by a fixed length independent from any input database. That is, the underlying $\Sigma_{\text {Research }}$ satisfies the so-called BDDP property, from which we know that the query answering under $\Sigma_{\text {Research }}$ is not only decidable, but also first-order rewritable (Calì, Gottlob, and Lukasiewicz 2012).

Main contributions of this paper are summarised here:

1. We define notations of derivation paths and derivation trees for query answering over TGDs (existential rules), and provide a precise characterisation for the traditional TGDs chase procedure through the corresponding derivation tree (Section 3).

2. Based on the concept of derivation paths, we introduce a new class called loop restricted (LR) TGDs, which are TGDs with certain restrictions on the loops embedded in the underlying rule set (Section 4).

3. Under our derivation tree framework, we show that the conjunctive query answering (CQA) under LR TGDs satisfies a property called bounded derivation tree depth property (BDTDP). We further prove that BDTDP implies the well-known bounded derivation-depth property (BDDP). This result implies that conjunctive query answering under LR TGDs is not only decidable but also first-order rewritable (Section 4).

4. We further extend LR TGDs to generalised loop restricted (GLR) TGDs, and prove that the class of GLR TGDs is also first-order rewritable and contains most of other firstorder rewritable TGD classes discovered in the literature so far (Sections 5 and 6).

\footnotetext{
${ }^{1}$ In general, $\operatorname{projDept}(X, Y, Z)$ means that staff $X$ from department $Y$ undertakes a project registered in department $Z$.
}

\section{Preliminaries}

In this section, we introduce necessary notions and definitions we will need through out this paper.

Databases and queries. We define the following pairwise disjoint (countably infinite) sets of symbols: a set $\Gamma$ of constants, which constitute the domain of databases, a set $\Gamma_{N}$ of labeled nulls that will be used as "fresh" Skolem terms as placeholders for unknown values, and a set $\Gamma_{V}$ of regular variables. For convenience, we usually use $a, b, c, \cdots$ to denote constants, $\mathrm{n}, \mathrm{n}^{\prime}, \mathrm{n}^{\prime \prime} \cdots$ to denote nulls, and $X, Y, Z, \cdots$ to denote variables. Note that different nulls may also represent the same value. We assume a lexicographic order on $\Gamma \cup \Gamma_{N}$, with every symbol in $\Gamma_{N}$ following all symbols in $\Gamma$. We use $\mathbf{X}$ to denote a sequence of variables $X_{1}, \cdots, X_{n}$, where $n \geq 0$. Sometimes, we also represent such $\mathbf{X}$ as a $n$ ary tuple of variables $\left(X_{1}, \cdots, X_{n}\right)$. A similar notion also applies to nulls.

A relational schema $\mathcal{R}$ is a finite set of relation symbols (or predicates). A term is a constant, null or variable. An atom has the form $p\left(t_{1}, \cdots, t_{n}\right)$, where $p$ is an $n$-ary predicate, and $t_{1}, \ldots, t_{n}$ are terms. We denote by $|p|$ and $\operatorname{dom}(p)$ as $p$ 's arity and the set of all its terms respectively. The latter notion is naturally extended to sets of atoms and conjunctions of atoms. A conjunction of atoms is often identified with the set of all its atoms.

A database $D$ for a relational schema $\mathcal{R}$ is a finite set of atoms with predicates from $\mathcal{R}$ and constants from $\Gamma$. That is, $\operatorname{dom}(D) \subseteq \Gamma$. We also use $\operatorname{pred}(D)$ to denote the set of all predicates occurring in $D$. An instance $I$ for a relational schema $\mathcal{R}$ is a (possibly infinite) set of atoms with predicates from $\mathcal{R}$ and terms from $\Gamma \cup \Gamma_{N}$. Clearly, each database $D$ for $\mathcal{R}$ may be viewed as a special form of instance, and further, it can be extended to an instance $I$ such that $D \subseteq I$ and $\operatorname{pred}(I)=\mathcal{R}$.

A homomophism from a set of atoms $\mathbf{A}$ to a set of atoms $\mathbf{A}^{\prime}$ is a mapping $h: \Gamma \cup \Gamma_{N} \cup \Gamma_{V} \rightarrow \Gamma \cup \Gamma_{N} \cup \Gamma_{V}$, such that (i) if $t \in \Gamma$, then $h(t)=t$; (ii) if $t \in \Gamma_{N}$, then $h(t) \in \Gamma \cup \Gamma_{N}$; and (iii) if $p\left(t_{1}, \cdots, t_{n}\right) \in \mathbf{A}$, then $p\left(h\left(t_{1}\right), \cdots, h\left(t_{n}\right)\right) \in \mathbf{A}^{\prime}$. Let $\mathbf{T}$ be the set of all terms occurring in $\mathbf{A}$. The restriction $h^{\prime}$ of $h$ to $\mathbf{S} \subseteq \mathbf{T}$, denoted as $h^{\prime}=\left.h\right|_{\mathbf{S}}$, is simply the subset of $h: h^{\prime}=\{t \rightarrow h(t) \mid t \in$ $\mathbf{S}\}$. Here we also call $h$ is an extension of $h^{\prime}$ to $\mathbf{T}$.

A conjunctive query (CQ) $q$ of arity $n$ over a schema $\mathcal{R}$ has the form $p(\mathbf{X}) \leftarrow \exists \mathbf{Y} \varphi(\mathbf{X}, \mathbf{Y})$, where $\varphi(\mathbf{X}, \mathbf{Y})$ is a conjunction of atoms with the variables $\mathbf{X}$ and $\mathbf{Y}$ from $\Gamma_{V}$ and constants from $\Gamma$, but without nulls, and $p$ is an $n$-ary predicate not occurring in $\mathcal{R}$. We allow $\varphi(\mathbf{X}, \mathbf{Y})$ to contain equalities but no inequalities. When $\varphi(\mathbf{X}, \mathbf{Y})$ is just a single atom, then we say that the CQ $q$ is atomic. A Boolean Conjunctive Query (BCQ) over $\mathcal{R}$ is a $\mathrm{CQ}$ of zero arity. In this case, we can simply write a BCQ $q$ as $\exists \mathbf{Y} \varphi(\mathbf{Y})$. A $\mathrm{CQ}$ answering problem, or called CQA problem, defined to be the answer to a CQ $q$ with $n$ arity over an instance $I$, denoted as $q(I)$, is the set of all $n$-tuples $\mathbf{t} \in \Gamma^{n}$ for which there exists a homomorphism $h: \mathbf{X} \cup \mathbf{Y} \rightarrow \Gamma \cup \Gamma_{V}$ such

\footnotetext{
${ }^{2}$ Possibly these constants, nulls and variable are subscripted with indexes.
} 
that $h(\varphi(\mathbf{X}, \mathbf{Y})) \subseteq I$ and $h(\mathbf{X})=\mathbf{t}$. The answer to a BCQ is positive over $I$, denoted as $I \models q$, if \langle\rangle$\in q(I)$.

TGDs and conjunctive query answering (CQA). A tuplegenerating dependency (TGD) $\sigma$, also called existential rule, over a schema $\mathcal{R}$ is a first-order formula of the form

$$
\sigma: \forall \mathbf{X Y} \varphi(\mathbf{X}, \mathbf{Y}) \rightarrow \exists \mathbf{Z} \psi(\mathbf{X}, \mathbf{Z}),
$$

where $\mathbf{X} \cup \mathbf{Y} \cup \mathbf{Z} \subset \Gamma \cup \Gamma_{V}, \varphi$ and $\psi$ are conjunctions of atoms over $\mathcal{R}$. When there is no confusion, we usually omit the universal quantifiers from (1). In this case, we also use $\operatorname{head}(\sigma)$ and $\operatorname{body}(\sigma)$ to denote formulas $\exists \mathbf{Z} \psi(\mathbf{X}, \mathbf{Z})$ and $\varphi(\mathbf{X}, \mathbf{Y})$ respectively.

Let $I$ be an instance over $\mathcal{R}$. We say that $\sigma$ is satisfied in $I$, denoted as $I \models \sigma$, if whenever there is a homomorphism $h$ such that $h(\varphi(\mathbf{X}, \mathbf{Y})) \subseteq I$, then there exists an extension $h^{\prime}$ of $\left.h\right|_{\mathbf{X}}$ such that $h^{\prime}(\psi(\overline{\mathbf{X}}, \mathbf{Z})) \subseteq I$.

Given a database $D$, a (finite) set $\Sigma$ of TGDs and a CQ $q$ of arity $n$ over schema $\mathcal{R}$. The models of $D$ with respect to $\Sigma$, denoted as $\bmod (D, \Sigma)$, is the set of all instances $I$ such that $I \supseteq D$ and $I \models \Sigma$. Then a CQ answering problem, or called CQA problem, denoted as $\langle\mathcal{R}, D, \Sigma, q\rangle$, is described as follows: the answer to $q$ with respect to $D$ and $\Sigma$, denoted as ans $(q, D, \Sigma)$, is the set of all tuples: $\{\mathbf{t} \mid \mathbf{t} \in q(I)$, for each $I \in \bmod (D, \Sigma)\}$. When $q$ is a BCQ, the answer to $q$ is called positive if \langle\rangle$\in \operatorname{ans}(q, D, \Sigma)$. It is well known that the CQA problem and the problem of CQ containment under TGDs are LOGSPACE-equivalent, and hence, in the rest of this paper, we will only focus on the BCQA problem, because all complexity results can be carried over to other problems (Calì, Gottlob, and Pieris 2012).

The chase algorithm. Consider an instance $I$ and a TGD $\sigma$ of the form (1). We say that $\sigma$ is applicable to $I$ if there exists a homomorphism $h$ such that $h(\varphi(\mathbf{X}, \mathbf{Y})) \subseteq I$. The result of applying $\sigma$ to $I$ is an instance $I^{\prime}=I \cup h^{\prime}(\psi(\mathbf{X}, \mathbf{Z}))$, where $h^{\prime}$ is an extension of $\left.h\right|_{\mathbf{X}}$ such that for each $Z \in \mathbf{Z}$, $h^{\prime}(Z)$ is a "fresh" labeled null of $\Gamma_{N}$ not occurring in $I$, and following lexicographically all those in $I$. Then the oblivious TGD chase algorithm for a database $D$ and a set $\Sigma$ of TGDs consists of an exhaustive application of chase steps in a fair fashion, which leads to a collection of all instances $I^{\prime}$ generated as described above, denoted as chase $(D, \Sigma)$. Note that each instance of chase $(D, \Sigma)$ is a model of $D \cup \Sigma$.

The above chase rule gives rise to the so-called chase sequence. A chase sequence: $I_{0} \stackrel{\sigma_{i}, h_{i}}{\longrightarrow} I_{1}, \ldots, I_{k} \stackrel{\sigma_{k}, h_{k}}{\longrightarrow}$ $I_{k+1}$, denotes the sequence of applications of the TGD chase rule such that: (1) $I_{0}=D$; (2) for each $i \in$ $\{1, \ldots, k\}, I_{i} \stackrel{\sigma_{i}, h_{i}}{\longrightarrow} I_{i+1}$ denotes the instance $I_{i+1}=I_{i}$ $\cup\left\{h_{i}^{\prime}\left(\operatorname{head}\left(\sigma_{i}\right)\right)\right\}$ such that assuming $\sigma_{i}=\varphi(\mathbf{X}, \mathbf{Y}) \rightarrow$ $\exists \mathbf{Z} \psi(\mathbf{X}, \mathbf{Z})$, then $h_{i}^{\prime}$ is the extension of the homomorphism $h_{i}\left\lceil\mathbf{x}\right.$ such that $h_{i}(\varphi(\mathbf{X}, \mathbf{Y})) \subseteq I_{i}$. Then lastly, for $k \geq$ 1 , we denote by chase ${ }^{[k]}(D, \Sigma)$ as the resulting instance $I_{k}$ that is the result of the chase sequence: $I_{0} \stackrel{\sigma_{i}, h_{i}}{\longrightarrow} I_{1}, \ldots$, $I_{k-1} \stackrel{\sigma_{k-1}, h_{k-1}}{\longrightarrow} I_{k}$.

The notion level in a chase is defined inductively as follows (Calì, Gottlob, and Pieris 2012): (1 ) for an atom $\alpha \in$ $D$, we set $\operatorname{LEVEL}(\alpha)=0$; then inductively, (2) for an atom $\alpha$ $\in$ chase $(D, \Sigma)$ obtained via some chase step $I_{k} \stackrel{\sigma, \eta}{\longrightarrow} I_{k+1}$, we set $\operatorname{LEVEL}(\alpha)=\operatorname{MAX}(\{\operatorname{LEVEL}(\beta) \mid \beta \in \operatorname{body}(\sigma \eta)\})+$ 1. Then finally, for some given $k \in \mathbb{N}$, we set chase ${ }^{k}(D, \Sigma)$ $=\{\alpha \mid \alpha \in$ chase $(D, \Sigma)$ and $\operatorname{LEVEL}(\alpha) \leq k\}$. Intuitively, chase $^{k}(D, \Sigma)$ is the instance containing atoms that can be derived in a less than or equal to $k$ chase steps.

Given an atom $p(\mathbf{t})$ such that $\mathbf{t} \in\left(\Gamma \cup \Gamma_{N}\right)^{|\mathbf{t}|}$, we say that chase $(D, \Sigma)$ entails $p(\mathbf{t})$ (chase ${ }^{[k]}(D, \Sigma)$ entails $p(\mathbf{t})$ ), denoted chase $(D, \Sigma) \models p(\mathbf{t})$ (chase $^{[k]}(D, \Sigma) \models p(\mathbf{t})$, resp.), iff there exists some atom of the same relational symbol $p\left(\mathbf{t}^{\prime}\right) \in \operatorname{chase}(D, \Sigma)\left(p\left(\mathbf{t}^{\prime}\right) \in\right.$ chase $^{[k]}(D, \Sigma)$, resp. $)$ and a homomorphism $h: \mathbf{t} \longrightarrow \mathbf{t}^{\prime}$ such that $h(p(\mathbf{t}))=p\left(\mathbf{t}^{\prime}\right)$.

Theorem 1. (Cali, Gottlob, and Pieris 2012) Given a BCQ q over $\mathcal{R}$, a database $D$ for $\mathcal{R}$ and a set $\Sigma$ of TGD s over $\mathcal{R}$, $D \cup \Sigma \models q$ iff chase $(D, \Sigma) \models q$.

Definition 1 (BDDP). A class $\mathcal{C}$ of TGDs satisfies the bounded derivation-depth property (BDDP) if for each $B C Q$ $q$ over a schema $\mathcal{R}$, for every input database $D$ for $\mathcal{R}$ and for every set $\Sigma \in \mathcal{C}$ over $\mathcal{R}, D \cup \Sigma \models q$ implies that there exists some $k \geq 0$ which only depends on $q$ and $\Sigma$ such that $\operatorname{chase}^{k}(D, \Sigma) \models q$.

It has been shown that the BDDP implies the firstorder rewritability (Calì, Gottlob, and Lukasiewicz 2012, Calì, Gottlob, and Pieris 2012). Formally, the BCQA problem is first-order rewritable for a class $\mathcal{C}$ of sets of TGDs if for each $\Sigma \in \mathcal{C}$, and each BCQ $q$, there exists a first-order query $q_{\Sigma}$ such that $D \cup \Sigma \models q$ iff $D \models q_{\Sigma}$, for every input database $D$. In this case, we also simply say that the class $\mathcal{C}$ of TGDs is first-order rewritable.

\section{Derivation Paths and Derivation Trees}

First of all, to simplify our investigations, from now on, we will assume that for any given set $\Sigma$ of TGDs, each TGD $\sigma$ in $\Sigma$ is of a specific form: $\sigma$ has only one atom in the head where each existentially quantified variable occurs only once. That is, $\Sigma$ consists of the following rule:

$$
\sigma: \varphi(\mathbf{X}, \mathbf{Y}) \rightarrow \exists \mathbf{Z} p(\mathbf{X}, \mathbf{Z})
$$

Theorem 2. Let $q$ be a BCQ over $\mathcal{R}, D$ a database for $\mathcal{R}$ and $\Sigma$ a set of TGDs over $\mathcal{R}$. Then we have:

1. There exists a LOGSPACE construction of an atomic BCQ $q^{\prime}$ and a set of TGDs $\Sigma^{\prime}$ of schema $\mathcal{R}^{\prime} \supseteq \mathcal{R}$, where $\left|\operatorname{head}\left(\sigma^{\prime}\right)\right|=1$ for each $\sigma^{\prime} \in \Sigma^{\prime}$, such that $D \cup \Sigma \models q$ iff $D \cup \Sigma^{\prime} \models q^{\prime}$ (Cali, Gottlob, and Pieris 2012).

2. If $\Sigma^{\prime}$ satisfies BDDP then $\Sigma$ also satisfies $B D D P$.

Under Theorem 2, it is clear that considering such special TGDs of the form (2) as well as the atomic BCQ $\exists \mathbf{Z} p(\mathbf{Z})$ will be sufficient, in the sense that all results related to these forms of TGDs and atomic BCQ can be carried over to the general case. So in the rest of this paper, we will only focus on these forms of TGDs and atomic BCQ in our study.

\section{Comparability and derivation paths}

Let $\mathbf{t}=\left(t_{1}, \cdots, t_{1}\right)$ and $\mathbf{t}^{\prime}=\left(t_{1}^{\prime}, \cdots, t_{n}^{\prime}\right)$ be two tuples of terms. We say that $\mathrm{t}$ and $\mathrm{t}^{\prime}$ are type comparable if $\mathrm{t}$ and $\mathrm{t}^{\prime}$ 
satisfy the following conditions: for each $i(1 \leq i \leq k)$, (1) constant $c \in \Gamma, t_{i}=c$ iff $t_{i}^{\prime}=c$; (2) $t_{i} \in \Gamma_{V}$ iff $\overline{t_{i}^{\prime}} \in \Gamma_{V}$; and (3) $t_{i} \in \Gamma_{N}$ iff $t_{i}^{\prime} \in \Gamma_{N}$. Intuitively, two tuples $\mathbf{t}$ and $\mathbf{t}^{\prime}$ are type comparable if each position between the two tuples agrees on the type of term they contain, i.e., constants are mapped to (the same) constants, variables to variables and labeled nulls into labeled nulls.

Definition 2 (Position comparable tuples). Let $\mathbf{t}=$ $\left(t_{1}, \cdots, t_{n}\right)$ and $\mathbf{t}^{\prime}=\left(t_{1}^{\prime}, \cdots, t_{n}^{\prime}\right)$ be two tuples of terms of length $n$. We say that $\mathbf{t}$ and $\mathbf{t}^{\prime}$ are position comparable (or simply called comparable), denoted as $\mathbf{t} \sim \mathbf{t}^{\prime}$, if $\mathbf{t}$ and $\mathbf{t}^{\prime}$ satisfy the following conditions:

1. $\mathbf{t}$ and $\mathbf{t}^{\prime}$ are type comparable;

2. for each pair $(i, j)(1 \leq i<j \leq n), t_{i}=t_{j}$ iff $t_{i}^{\prime}=t_{j}^{\prime}$;

3. $t \in\left(\mathbf{t} \cap \mathbf{t}^{\prime}\right), t_{i}=t$ iff $t_{i}^{\prime}=t(1 \leq i \leq n)$.

We also use $\mathbf{t}_{\mathbf{1}} \nsim \mathbf{t}_{2}$ if it is not the case that $\mathbf{t}_{\mathbf{1}} \sim \mathbf{t}_{2}$.

Under Definition 2, we have $\left(X, X^{\prime}, \mathrm{n}\right) \sim\left(Z, Y, \mathrm{n}^{\prime}\right)$, but $\left(\mathrm{n}, \mathrm{n}, \mathrm{n}^{\prime}, Z\right) \nsim\left(\mathrm{n}, \mathrm{n}^{\prime}, \mathrm{n}^{\prime}, W\right)$, because in the latter, the null patterns in the first three positions of the two tuples are not "comparable".

Let $X$ be a variable from $\Gamma_{V}$, and $t$ a term from $\Gamma \cup \Gamma_{N}$ $\cup \Gamma_{V}$. A binding is an expression of the form $X / t$. In this case, we also say that $t$ is a binding of variable $X$. A substitution $[\mathbf{X} / \mathbf{t}]$ is a finite set of bindings containing at most one binding for each variable from $\mathbf{X}$. For a given tuple of terms $\mathbf{t}$, we apply a substitution $\theta$ to $\mathbf{t}$ and obtain a different tuple of terms, denoted as $\mathbf{t} \theta$. For example, $(X, Y, \mathrm{n}, W)\left[X / \mathrm{n}^{\prime}, Y / Y, W / Z\right]=\left(\mathrm{n}^{\prime}, Y, \mathrm{n}, Z\right)$. For a quantifier-free formula $\varphi(\mathbf{X})$ and a substitution $\theta=[\mathbf{X} / \mathbf{t}]$, applying $\theta$ to $\varphi(\mathbf{X})$, i.e., $\varphi(\mathbf{X}) \theta$, will result in formula $\varphi(\mathbf{t})$ which is obtained from $\varphi(\mathbf{X})$ by replacing each free variable $X$ by its corresponding binding from $\varphi(\mathbf{X})$.

Now we define how a substitution is applied to an existential rule $\sigma$. We extend a substitution to existentially quantified variables. We say that substitution $\theta=[\mathbf{X} / \mathbf{t}]$ is applicable to $\sigma$ if the arities of $\mathbf{X}$ in $\theta$ match the arities of the tuples of all universally and existentially quantified variables in $\sigma$, respectively. We may write a substitution applicable to $\sigma$ as the form: $\theta=\left[\mathbf{X} / \mathbf{t}_{1}, \mathbf{Y} / \mathbf{t}_{2}, \mathbf{Z} / \mathbf{n}\right]$. Then by applying $\theta$ to rule $\sigma$ of the form (2), we will obtain a rule of the following form:

$$
\sigma \theta: \varphi\left(\mathbf{t}_{1}, \mathbf{t}_{2}\right) \rightarrow p\left(\mathbf{t}_{1}, \mathbf{n}\right)
$$

Definition 3 (Derivation path). Let $\Sigma$ be a set of TGDs. $A$ derivation path $P$ of $\Sigma$ is a finite sequence of pairs of an atom and a rule:

$$
\left(\alpha_{1}, \rho_{1}\right), \cdots,\left(\alpha_{n}, \rho_{n}\right)
$$

such that

- for each $1 \leq i \leq n, \alpha_{i}=\operatorname{head}\left(\rho_{i}\right)$;

- for each $1 \leq i \leq n, \rho_{i}=\sigma_{i} \theta_{i}$ for some $\sigma_{i} \in \Sigma$ and substitution $\theta_{i}$;

- for each $1 \leq i<n, \alpha_{i+1} \in \operatorname{body}\left(\rho_{i}\right)$;

- for each $1 \leq i \leq n$, if a null $\mathrm{n} \in \operatorname{head}\left(\alpha_{i}\right)$ is introduced due to the elimination of existentially quantified variable, then this $\mathrm{n}$ must not occur in $\rho_{j}$, for all $j \in\{i+1, \ldots, n\}$.
Example 2. Consider a set $\Sigma$ of TGDs consisting of two rules:

$$
\begin{aligned}
& \sigma_{1}: r(X, Y, Z) \rightarrow s(Y, X), \\
& \sigma_{2}: s(X, Y) \rightarrow \exists Z \exists W r(Y, Z, W) .
\end{aligned}
$$

The following are three different derivation paths of $\Sigma$ :

$$
\begin{aligned}
P_{1}: \quad & \left(s\left(\mathrm{n}_{1}, Y_{1}\right), \sigma_{1}\left[X / Y_{1}, Y / \mathrm{n}_{1}, Z / \mathrm{n}_{2}\right]\right), \\
& \left(r\left(Y_{1}, \mathrm{n}_{1}, \mathrm{n}_{2}\right), \sigma_{2}\left[X / X_{1}, Y / Y_{1}, Z / \mathrm{n}_{1}, W / \mathrm{n}_{2}\right]\right), \\
& \left(s\left(X_{1}, Y_{1}\right), \sigma_{1}\left[X / Y_{1}, Y / X_{1}, Z / Z_{1}\right]\right), \\
P_{2}: \quad & \left(r\left(X_{2}, \mathrm{n}_{1}, \mathrm{n}_{2}\right), \sigma_{2}\left[X / \mathrm{n}_{3}, Y / X_{2}, Z / \mathrm{n}_{1}, W / \mathrm{n}_{2}\right]\right), \\
& \left(s\left(\mathrm{n}_{3}, X_{2}\right), \sigma_{1}\left[X / X_{2}, Y / \mathrm{n}_{3}, Z / \mathrm{n}_{4}\right]\right), \\
P_{3}: \quad & \left(r\left(X_{2}, \mathrm{n}_{1}, \mathrm{n}_{2}\right), \sigma_{2}\left[X / \mathrm{n}_{3}, Y / X_{2}, Z / \mathrm{n}_{1}, W / \mathrm{n}_{2}\right]\right), \\
& \left(s\left(\mathrm{n}_{3}, X_{2}\right), \sigma_{1}\left[X / X_{2}, Y / \mathrm{n}_{3}, Z / \mathrm{n}_{4}\right]\right), \\
& \left(r\left(X_{2}, \mathrm{n}_{3}, \mathrm{n}_{4}\right), \sigma_{2}\left[X / X_{1}, Y / X_{2}, Z / \mathrm{n}_{3}, W / \mathrm{n}_{4}\right]\right) .
\end{aligned}
$$

Definition 4 (Generalising comparability relation). We generalise the comparability relation $\sim$ defined earlier as follows.

1. Let $\sigma \in \Sigma$, and $\theta=[\mathbf{X} / \mathbf{t}, \mathbf{Z} / \mathbf{n}]$ and $\theta^{\prime}=\left[\mathbf{X} / \mathbf{t}^{\prime}, \mathbf{Z} / \mathbf{n}^{\prime}\right]$ be two substitutions applicable to $\sigma$. We say that $\sigma \theta$ and $\sigma \theta^{\prime}$ are comparable, denoted as $\sigma \theta \sim \sigma \theta^{\prime}$, if $\mathbf{t n} \sim \mathbf{t}^{\prime} \mathbf{n}^{\prime}$.

2. Let $P$ be a derivation path of $\Sigma$ of the form (4), we use $|P|$ to denote its length. Furthermore, suppose $\left(\alpha_{i}, \rho_{i}\right)$ and $\left(\alpha_{j}, \rho_{j}\right)$ are two elements of $P$, we say that $\left(\alpha_{i}, \rho_{i}\right)$ and $\left(\alpha_{j}, \rho_{j}\right)$ are comparable, denoted as $\left(\alpha_{i}, \rho_{i}\right) \sim\left(\alpha_{j}, \rho_{j}\right)$, if $\rho_{i} \sim \rho_{j}$ (note that this implies $\sigma_{i}=\sigma_{j}$ ).

3. Let $P=\left(\left(\alpha_{1}, \rho_{1}\right),\left(\alpha_{2}, \rho_{2}\right), \cdots\right)$ and $P^{\prime}=$ $\left(\left(\alpha_{1}^{\prime}, \rho_{1}^{\prime}\right),\left(\alpha_{2}^{\prime}, \rho_{2}^{\prime}\right), \cdots\right)$ be two derivation paths of $\Sigma$. $P$ and $P^{\prime}$ are comparable, denoted as $P \sim P^{\prime}$, if $|P|=\left|P^{\prime}\right|$ and for each $i(1 \leq i \leq|P|),\left(\alpha_{i}, \rho_{i}\right) \sim\left(\alpha_{i}^{\prime}, \rho_{i}^{\prime}\right)$.

It is easy to observe that $\sim$ defined in Definition 4 is an equivalence relation. Although a derivation path may be infinitely long, the following result ensures that for any derivation path, it is sufficient to only consider its finite fragment.

Proposition 1 (Derivation path length bound). Let $\Sigma$ be a set of TGDs. Then there exists a natural number $N$ such that for every derivation path $P$ of the form (4), if $|P|>N$ then there exists $i, j(1 \leq i<j \leq|P|)$ such that $\left(\alpha_{i}, \rho_{i}\right) \sim$ $\left(\alpha_{j}, \rho_{j}\right)$.

\section{Derivation trees}

Definition 5 (Derivation tree). Given a set $\Sigma$ of TGDs. $A$ derivation tree of $\Sigma$, denoted as $T(\Sigma)$, is a finite tree $(N, E, \lambda)$, with nodes $N$, edges $E$ and labeling function $\lambda$, such that:

1. The nodes of $T(\Sigma)$ have labels of the form $(\alpha, \rho)$, where $\rho=\sigma \theta$ for some $\sigma \in \Sigma$ and $\theta$ a substitution, and $\operatorname{head}(\rho)=\alpha$;

2. For any node $v$ labeled by $(\alpha, \rho)$ of $T(\Sigma)$, let $\alpha_{1}, \cdots, \alpha_{n}$ be atoms in body $(\rho)$, then $(\alpha, \rho)$ has $n$ children $v_{1}, \ldots, v_{n}$ labeled with $\left(\alpha_{1}, \rho_{1}\right), \cdots,\left(\alpha_{n}, \rho_{n}\right)$, respectively, such that for each $i \in\{1, \ldots, n\}, \rho_{i}=\sigma_{i} \theta_{i}$ for some $\sigma_{i} \in \Sigma$ and $\theta_{i}$ a substitution, and head $\left(\rho_{i}\right)=\alpha_{i}$;

3. For any node $v$ labeled with $(\alpha, \rho)$ in $T(\Sigma)$, all "fresh" nulls occurring in $\alpha$, that are introduced through the substitutions in $\rho$, must not occur in any labels of a descendant node of $v$; 
4. If node $v$ labeled with $(\alpha, \rho)$ is a leaf of $T(\Sigma)$, then there does not exist any null $\mathrm{n}$ appearing in body $(\rho)$.

A path $P$ in $T(\Sigma)$, denoted as $P \in T(\Sigma)$, is a derivation path in $T(\Sigma)$ starting from the root and ending at a leaf. We define depth $(T(\Sigma))=\max (\{|P| \mid P \in T(\Sigma)\}$ to be the depth of $T(\Sigma)$. By $\operatorname{root}(T(\Sigma))$, leafNodes $(T(\Sigma))$ and nodes $(T(\Sigma)$ ), we denote the root node, leaf nodes and all nodes of $T(\Sigma)$, respectively. Also, given some node $v$ of $T(\Sigma)$, we denote by childNodes $(v, T(\Sigma)$ ) (or just childNodes $(v)$ when clear from the context) as the child nodes of $v$ under the tree $T(\Sigma)$. Lastly, we use $\mathcal{T}(\Sigma)$ to denote the set of all derivation trees of $\Sigma$.

According to Definition 5, each path of a derivation tree is a derivation path. Also, since $\Sigma$ can have an infinite number of possible derivation paths due to possibly arbitrary number of repetitions of path fragments within a path (i.e., a "loop"), $\mathcal{T}(\Sigma)$ may contain an infinite number of derivation trees.

Definition 6 (Derivation tree instantiation). Let $\Sigma$ be a set of TGDs, $D$ a database over schema $\mathcal{R}$, and $T(\Sigma)=$ $(N, E, \lambda)$ a derivation tree of $\Sigma$. Then we obtain a tree $T^{\prime}$ $=\left(N^{\prime}, E^{\prime}, \lambda^{\prime}\right)$ from $T(\Sigma)$, where $N \subseteq N^{\prime}$, as follows:

1. For each leaf node $v$ in $T(\Sigma)$, where $\lambda(v)=(\alpha, \rho)$, do:

(a) Set $\lambda^{\prime}(v)=\left(\alpha^{\prime}, \rho^{\prime}\right)$ in the tree $T^{\prime}$, where $\alpha^{\prime}=$ head $\left(\rho^{\prime}\right)$, where $\rho^{\prime}=\rho \theta$ for some substitution $\theta$ and $\operatorname{body}\left(\rho^{\prime}\right) \subseteq D$;

(b) For each atom $\beta \in \operatorname{body}\left(\rho^{\prime}\right) \subseteq D$ with $\rho^{\prime}$ as mentioned above, add a node $v^{\prime}$ in $N^{\prime}$ and set $\lambda^{\prime}\left(v^{\prime}\right)=(\beta, \beta)$ and corresponding edge $\left\langle v^{\prime}, v\right\rangle$ in $E$ so that $v^{\prime}$ is a leaf node (so now making $v$ a non-leaf node);

2. For a node $v$ such that $\lambda(v)=(\alpha, \rho)$, and where all the label of its children have been replaced as in 1 above (i.e., through “ ' $\lambda^{\prime}$ ”), set $\lambda^{\prime}(v)=\left(\alpha^{\prime}, \rho^{\prime}\right)$, where $\rho^{\prime}=\rho \theta^{\prime}$ for some substitution $\theta^{\prime}$ such that for each atom $p(\mathbf{t}) \in$ body $\left(\rho^{\prime}\right)$, either $p(\mathbf{t}) \in D$ or there exists a child node $v^{\prime}$ of $v$ such that $\lambda^{\prime}\left(v^{\prime}\right)=\left(\alpha^{*}, \rho^{*}\right)$, where $p(\mathbf{t})=\alpha^{*}$;

3. Continue 2 , until no node can be further relabled.

$T^{\prime}$ is called an instantiation of $T(\Sigma)$ on $D$, denoted as $T(D, \Sigma)$, if it does not contain any variables occurring in $T(\Sigma)$. Similarly to the case of derivation tree, we use depth $(T(D, \Sigma))$ and $\operatorname{root}(T(D, \Sigma))$ to denote the depth and root node of $T(D, \Sigma)$, respectively. Finally, by $\mathcal{T}(D, \Sigma)$, we denote the set of all instantiations on $D$ for all derivation trees in $\mathcal{T}(\Sigma)$.

For convenience from here on and when it is clear from the context, we will mostly refer to a node by its actual label, e.g., a node $v \in N$ where $\lambda(v)=(\alpha, \rho)$ is simply refered to as $(\alpha, \rho)$.

We say that an atom $p(\mathbf{t})$ is supported by $T(D, \Sigma)$, denoted as $T(D, \Sigma) \models p(\mathbf{t})$, if $\lambda(\operatorname{root}(T(D, \Sigma)))=(\alpha, \rho)$ where $\alpha=p(\mathbf{s})$, and there is a homomorphism $h$ such that $h(p(\mathbf{t}))=p(\mathbf{s})$. The following result reveals an important relationship between the chase and derivation trees.

Theorem 3. Let $\Sigma$ be a set of TGDs, $D$ a database over schema $\mathcal{R}$, and q a $B C Q$ query $\exists \mathbf{Z} p(\mathbf{Z})$. Then chase $(D, \Sigma) \models q$ iff there exist an instantiation $T(D, \Sigma)$ for some derivation tree $T(\Sigma)$ and a substitution $\theta$, such that
$T(D, \Sigma) \models p(\mathbf{t})$, where $\mathbf{t}$ is a tuple of terms from $\Gamma$ of the same length as $\mathbf{Z}$, and $\mathbf{t} \theta=\mathbf{Z}$.

Proof. (“””) We prove this direction by first providing the following lemma.

Lemma 1. Given an instantiated derivation tree $T(D, \Sigma)$ $=(N, E, \lambda)$ with nodes $N$, edges $E$ and labeling function $\lambda$, of $\Sigma$ under a database $D$, there exists a homomorphism $\mu: \operatorname{nodes}(T(D, \Sigma)) \longrightarrow$ chase $^{[N]}(D, \Sigma)$, where $N$ $\leq|\operatorname{nodes}(T(D, \Sigma))|-\mid$ leafNodes $(T(D, \Sigma)) \mid$, such that the following conditions are satisfied:

1. For each $v \in$ leafNodes $(T(D, \Sigma))$ such that $\lambda(v)=(\alpha, \alpha)$, $\mu(v)=\alpha \in D ;$

2. For each $v \in \operatorname{nodes}(T(D, \Sigma))$ such that $\lambda(v)=(\alpha, \rho)$, $\operatorname{child}(v)=\left\{v_{1}, \ldots, v_{1}\right\}, \rho=\sigma \theta$ for some substitution $\theta$, and $\sigma=\varphi(\mathbf{X}, \mathbf{Y}) \rightarrow \exists \mathbf{Z} p(\mathbf{X}, \mathbf{Z}) \in \Sigma$, there exists a homorphism $h$ such that $h(\varphi(\mathbf{X}, \mathbf{Y})) \subseteq\left\{\mu\left(v_{1}\right), \ldots, \mu\left(v_{n}\right)\right\}$ and extension $h^{\prime}$ of $h \uparrow_{\mathbf{X}}$ where $\mu(v)=h^{\prime}(p(\mathbf{X}, \mathbf{Z}))$;

3. For each $v \in \operatorname{nodes}(T(D, \Sigma))$ such that $\lambda(v)=(\alpha, \rho)$ and $\alpha=p(\mathbf{t})$, if $\mu(v)=q\left(\mathbf{t}^{\prime}\right)$ then we have that $p(\mathbf{t}) \theta=q\left(\mathbf{t}^{\prime}\right)$

for some substitution $\theta$.

4. For each $v_{1}, v_{2} \in \operatorname{nodes}(T(D, \Sigma))$ such that $\lambda\left(v_{1}\right)=\lambda\left(v_{2}\right)$ (i.e., $v_{1}$ and $v_{2}$ have the same label), then we also have that $\mu\left(v_{1}\right)=\mu\left(v_{2}\right)$.

Proof (Sketch). We show the existence of such a homomorphism $\mu$ by induction on the depth of the tree $T(D, \Sigma)$ starting from the leaf nodes (i.e., the nodes labeld by the database facts) going up to the root node labeled by $(\alpha, \rho)$.

Then from Lemma 1 since $T(D, \Sigma) \models p(\mathbf{t})$, then assuming that $\lambda(\operatorname{root}(T(D, \Sigma)))=(\alpha, \rho)$ such that $\alpha=$ $r(\mathbf{s})$, we have from the definition of "instantiated tree supportedness" of an atom that $h(r(\mathbf{t}))=r(\mathbf{s})$ for some homomorphism $h: \mathbf{t} \longrightarrow \mathbf{s}$. Then because we have that $\mu(r(\mathbf{s}))=r\left(\mathbf{t}^{\prime}\right)$ for some atom $r\left(\mathbf{t}^{\prime}\right) \in$ chase $^{[N]}(D, \Sigma)$, where $N \leq|\operatorname{nodes}(T(D, \Sigma))|-\mid$ leafNodes $(T(D, \Sigma)) \mid$ and $\mu: \operatorname{nodes}(T(D, \Sigma)) \longrightarrow$ chase $(D, \Sigma)$ the "bounding number" and homomorphism defined in Lemma1, respectively, then we also have from Lemma 1 that $r(\mathbf{s}) \theta=r\left(\mathbf{t}^{\prime}\right)$ for some substitution $\theta$. Therefore, with $h^{\prime}=\theta \circ h$, then we have that $h^{\prime}(p(\mathbf{t}))=q\left(\mathbf{t}^{\prime}\right) \in$ chase $^{[N]}(D, \Sigma)$, which implies that chase $^{[N]}(D, \Sigma) \models p(\mathbf{t})$.

(“ऍ”) Assume chase ${ }^{[N]}(D, \Sigma) \models p(\mathbf{t})$ for some atom $p(\mathbf{t})$ and $N \geq 1$. Then by the definition of chase ${ }^{[N]}(D, \Sigma)$ $\models p(\mathbf{t})$, there exists some atom $p\left(\mathbf{t}^{\prime}\right) \in$ chase $^{[N]}(D, \Sigma)$ and homomorphism $h: \mathbf{t}^{\prime} \longrightarrow \mathbf{t}$ such that $h(p(\mathbf{t}))=p\left(\mathbf{t}^{\prime}\right)$. Thus, there exists some finite chase sequence $I_{0} \stackrel{\sigma_{0}, h_{0}}{\longrightarrow} I_{1}$, $\ldots, I_{N-1} \stackrel{\sigma_{N}, h_{N}}{\longrightarrow} I_{N}$ such that $p\left(\mathbf{t}^{\prime}\right) \in I_{N}$. Let us assume without loss of generality that for $i \in\{1, \ldots, N-1\}$, there does not exists another atom $p\left(\mathbf{t}^{\prime \prime}\right) \in I_{i}$ such that $h(p(\mathbf{t}))$ 
$=p\left(\mathbf{t}^{\prime \prime}\right)$. Then based on the sequences of TGDs $\sigma_{i}$ and homomorphisms $h_{i}$ that made $\sigma_{i}$ applicable to $I_{i}$, we can construct an instantiated derivation tree $T(D, \Sigma)$ as follows:

1. Let $\operatorname{root}(T(D, \Sigma))$ be labeled with $\left(p\left(\mathbf{t}^{\prime}\right), \sigma_{N} \theta_{N}\right)$, where $\theta_{N}$ is the corresponding substitution for $h_{N}$ and its extension $h_{N}^{\prime}$;

2. For each atom $\alpha \in$ chase $^{[N]}(D, \Sigma)$ either :

- add a node $v$ with label $(\alpha, \alpha)$, if $\alpha \in D$, otherwise

- add a node $v$ with label $(\alpha, \rho)$, where $\alpha=\operatorname{head}(\rho)$, $\rho=\sigma_{i} \theta_{i}$ and $\theta_{i}$ the corresponding substitution for $h_{i}$ and its "extension" $h_{i}^{\prime}$.

3. For each node $v$ with label $(\alpha, \rho)$ such that $\rho=\sigma \theta$, for some $\sigma \in \Sigma$ and substitution $\theta$, and $\operatorname{body}(\rho)=$ $\left\{\alpha_{1}, \ldots, \alpha_{n}\right\}$ then for $i \in\{1, \ldots, n\}$, add an edge $\left(v, v_{i}\right)$ such that either:

- $v_{i}$ is labled with $\left(\alpha_{i}, \alpha_{i}\right)$, if $\alpha_{i} \in D$, otherwise

- $v_{i}$ is labled with $\left(\alpha_{i}, \rho_{i}\right)$, such that $\alpha_{i}=\operatorname{head}\left(\rho_{i}\right)$, $\rho_{i}=\sigma_{j} \theta_{j}, \theta_{j}$ the corresponding subtitution for $h_{j}$ (and corresponding extension $h_{j}^{\prime}$ ) and $I_{j} \stackrel{\sigma_{j}, h_{j}}{\longrightarrow} I_{j+1}$ is the first chase step that derived $\alpha_{i}$.

Then it is not too difficult to see that the above construction for $T(D, \Sigma)$ is in fact an instantiated derivation tree and where $N \leq|\operatorname{nodes}(T(D, \Sigma))|-|\operatorname{leafNodes}(T(D, \Sigma))|$. (i.e., recall that $p\left(\mathbf{t}^{\prime}\right) \in I_{N}$ such that $I_{N-1} \stackrel{\sigma_{N}, h_{N}}{\longrightarrow} I_{N}$ is the first chase step that derived $p\left(\mathbf{t}^{\prime}\right)$ ). Therefore, because $h(p(\mathbf{t}))=p\left(\mathbf{t}^{\prime}\right)$ for some homomorphism $h: \mathbf{t} \longrightarrow$ $\mathbf{t}^{\prime}$ (i.e., recall that chase $(D, \Sigma) \models p(\mathbf{t})$ and $p\left(\mathbf{t}^{\prime}\right) \in$ chase such that $\left.h(p(\mathbf{t}))=p\left(\mathbf{t}^{\prime}\right)\right)$ and since, assuming that $(\alpha, \rho)$ $=\operatorname{root}(T(D, \Sigma))$, we have that $p\left(\mathbf{t}^{\prime}\right)=\alpha$ from the construction of $T(D, \Sigma)$, then we clearly have that $T(D, \Sigma) \models p(\mathbf{t})$ through the same "witnessing" homomorphism $h$.

\section{Loop Restricted (LR) TGDs}

Theorem 3 shows that derivation trees provide a precise characterisation for the chase procedure. Therefore, the query answering against a set of TGDs together with an input database can be achieved by computing and checking the corresponding instantiation of the underlying derivation tree. However, since the derivation tree for a given set of TGDs may be of an arbitrary depth, this process is generally undecidable.

In this section, we will define a new class of TGDs, named loop restricted (LR) TGDs, such that the depth of all derivation trees for this type of TGDs is always bounded in some sense. From this result, we will further prove that LR TGDs satisfy the bounded derivation-depth property (BDDP) (Calì, Gottlob, and Pieris 2012).

Definition 7 (Loop pattern). Let $P=\left(\left(\alpha_{1}, \rho_{1}\right), \cdots\right.$, $\left.\left(\alpha_{n}, \rho_{n}\right)\right)$ be a derivation path as defined in Definition 3. Then $P$ is a loop pattern if $\left(\alpha_{1}, \rho_{1}\right) \sim\left(\alpha_{n}, \rho_{n}\right)$ and $\left(\alpha_{i}, \rho_{i}\right) \not\left(\alpha_{j}, \rho_{j}\right)$ for any other $i, j(1<i, j<n)$.
Let $L$ be a loop pattern as defined in Definition 7. For each pair $\left(\alpha_{i}, \rho_{i}\right)$ in $L$ where $1 \leq i<n$, an atom $\beta \in \operatorname{body}\left(\rho_{i}\right)$ is called recursive atom if $\beta=\alpha_{i+1}$ for $\left(\alpha_{i+1}, \rho_{i+1}\right)$.

Example 3. Example 2 continued. It is easy to see that derivation paths $P_{1}$ and $P_{3}$ are loop patterns, while $P_{2}$ is not. Furthermore, $P_{1}$ and $P_{3}$ are the only two different loop patterns of the given $\Sigma$, considering that for all other loop patterns $P$, it will be either $P \sim P_{1}$ or $P \sim P_{3}{ }^{3}$.

Proposition 2. Given a finite set $\Sigma$ of TGDs, $\Sigma$ only has a finite number of loop patterns under equivalence relation $\sim$.

\section{Restricted loop patterns}

Example 4. Consider Example 1 in Introduction again. Here we simplify $\Sigma_{\text {Research }}$ by removing $\sigma_{3}$ and renaming predicates in $\sigma_{1}$ and $\sigma_{2}$. Note that such change will not affect $\Sigma_{\text {Research }}$ 's original loop pattern.

$$
\begin{aligned}
& \sigma_{1}: q(X, Y) \rightarrow p(X), \\
& \sigma_{2}: p(X), r(X, Y), s(X, Y, Y) \rightarrow \exists W q(X, W) .
\end{aligned}
$$

We can verify that $\Sigma$ does not belong to any of currently known first-order rewritable TGDs classes.

Now we consider the derivation of atom $q\left(X_{1}, \mathrm{n}_{1}\right)$ from $\Sigma$. The following are two different derivation trees for $q\left(X_{1}, \mathrm{n}_{1}\right)$, and both involve recursive calls to $\sigma_{1}$ and $\sigma_{2}$ :

$T_{1}:$

$$
\begin{aligned}
w_{0}^{1} & =\left(\alpha_{0}^{1}, \rho_{0}^{1}\right) \\
& =\left(q\left(X_{1}, \mathrm{n}_{1}\right),\left[p\left(X_{1}\right), r\left(X_{1}, X_{2}\right), s\left(X_{1}, X_{2}, X_{2}\right) \rightarrow q\left(X_{1}, \mathrm{n}_{1}\right)\right]\right), \\
w_{1}^{1} & =\left(\alpha_{1}^{1}, \rho_{1}^{1}\right) \\
& =\left(p\left(X_{1}\right),\left[q\left(X_{1}, \mathrm{n}_{2}\right) \rightarrow p\left(X_{1}\right)\right]\right), \\
w_{2}^{1} & =\left(\alpha_{2}^{1}, \rho_{2}^{1}\right) \\
& =\left(q\left(X_{1}, \mathrm{n}_{2}\right),\left[p\left(X_{1}\right), r\left(X_{1}, X_{3}\right), s\left(X_{1}, X_{3}, X_{3}\right) \rightarrow q\left(X_{1}, \mathrm{n}_{2}\right)\right]\right) ; \\
T_{2}: & \\
w_{0}^{2} & =\left(\alpha_{0}^{2}, \rho_{0}^{2}\right) \\
& =\left(q\left(X_{1}, \mathrm{n}_{1}\right),\left[p\left(X_{1}\right), r\left(X_{1}, X_{2}\right), s\left(X_{1}, X_{2}, X_{2}\right) \rightarrow q\left(X_{1}, \mathrm{n}_{1}\right)\right]\right), \\
w_{1}^{2} & =\left(\alpha_{1}^{2}, \rho_{1}^{2}\right) \\
& =\left(p\left(X_{1}\right),\left[q\left(X_{1}, \mathrm{n}_{2}\right) \rightarrow p\left(X_{1}\right)\right]\right), \\
w_{2}^{2} & =\left(\alpha_{2}^{2}, \rho_{2}^{2}\right) \\
& =\left(q\left(X_{1}, \mathrm{n}_{2}\right),\left[p\left(X_{1}\right), r\left(X_{1}, X_{3}\right), s\left(X_{1}, X_{3}, X_{3}\right) \rightarrow q\left(X_{1}, \mathrm{n}_{2}\right)\right]\right), \\
w_{3}^{2} & =\left(\alpha_{3}^{2}, \rho_{3}^{2}\right) \\
& =\left(p\left(X_{1}\right),\left[q\left(X_{1}, X_{4}\right) \rightarrow p\left(X_{1}\right)\right]\right) .
\end{aligned}
$$

Intuitively, $T_{1}$ simply contains one derivation path $L_{1}=$ $w_{0}^{1} w_{1}^{1} w_{2}^{1}$ which is also a loop pattern, while $T_{2}$ 's loop pattern is $L_{2}=w_{1}^{2} w_{2}^{2} w_{3}^{2}$. If we consider all other derivation trees for atom $q\left(X_{1}, \mathrm{n}_{1}\right)$, it is not difficult to observe that all these trees are subsumed by either $T_{1}$ or $T_{2}$, in the sense that derivations illustrated in $T_{1}$ or $T_{2}$ sufficiently cover those illustrated in all other trees

$\Sigma$ presents an interesting case of satisfying the so-called bounded derivation tree depth property (BDTDP) (the definition will be given later). By examining the two loop patterns, we find that they can be split in such a way where all

${ }^{3}$ See Definition 4 for derivation path (loop pattern) comparability relation. 
variables in the recursive atoms are bounded by the variables occurring in the heads of all corresponding rules. This will make the derived atom in each derivation step from the corresponding derivation tree not rely on any new variables in recursive atoms.

Consider loop pattern $L_{1}$, for instance, for each pair $\left(\alpha_{i}^{1}, \rho_{i}^{1}\right)(i=0,1,2)$, we can split the $\operatorname{set} \operatorname{body}\left(\rho_{i}^{1}\right)$ of atoms in the body of $\rho_{i}^{1}$ into two disjoint parts body $\left(\rho_{i}^{1}\right)$ and $\operatorname{body}_{\mathbf{b}}\left(\rho_{i}^{1}\right)$, such that the common variables in $\alpha_{i}^{1} \cup$ $\operatorname{body}_{\mathrm{h}}\left(\rho_{i}^{1}\right)$ and body $\left(\rho_{i}^{1}\right)$ are exactly the common variable occurring in all $\alpha_{i}$, which is $X_{1}$, whilst the underlying recursive atoms in the loop pattern only occur in $\operatorname{body}_{\mathbf{b}}\left(\rho_{i}^{1}\right)$, i.e., $\alpha_{i+1}^{1} \in \operatorname{body}_{\mathrm{b}}\left(\rho_{i}^{1}\right)$ for $i=0,1$. We can do a similar separation for loop pattern $L_{2}$ as well. As will be showed next, it turns out that a set $\Sigma$ of TGDs having this feature always ensures BDTDP.

Now we are ready to formally define the notion of restricted loop patterns. Let $A$ be a set of atoms, we use $\operatorname{var}(A)$ to denote the set of all variables occurring in $A$.

Definition 8 (Loop restricted (LR) patterns). Let $\Sigma$ be a set of TGDs. $\Sigma$ is loop restricted (LR), if for each loop pattern $L=\left(\alpha_{1}, \rho_{1}\right) \cdots\left(\alpha_{n}, \rho_{n}\right)$ of $\Sigma$, L satisfies the following conditions: for each pair $\left(\alpha_{i}, \rho_{i}\right)$ in $L(1 \leq i<n)$, the set of atoms body $\left(\rho_{i}\right)$ can be separated into two disjoint parts body $\left(\rho_{i}\right)=$ body $_{\mathrm{h}}\left(\rho_{i}\right) \cup \operatorname{body}_{\mathrm{b}}\left(\rho_{i}\right)$, such that (1) $\operatorname{body}_{\mathrm{h}}\left(\rho_{i}\right) \cap \operatorname{body}_{\mathrm{b}}\left(\rho_{i}\right)=\emptyset$, (2) $\alpha_{i+1} \in \operatorname{body}_{\mathrm{b}}\left(\rho_{i}\right)$, and (3) $\operatorname{var}\left(\left\{\alpha_{i}\right\} \cup \operatorname{body}_{\mathrm{h}}\left(\rho_{i}\right)\right) \cap \operatorname{var}\left(\operatorname{body}_{\mathrm{b}}\left(\rho_{i}\right)\right)=\bigcap_{j=1}^{n} \operatorname{var}\left(\alpha_{j}\right)$.

Example 5. Example 4 continued. It is easy to see that loop patterns $L_{1}$ and $L_{2}$ in Example 4 satisfy the conditions of Definition 8. Furthermore, if we consider the derivation of atom $p(X)$ from $\Sigma$, the underlying loop patterns deduced from its derivations also satisfy the conditions of Definition 8. So $\Sigma$ is loop restricted.

\section{Main results}

Now we study the main properties of the new class LR TGDs. We first define a property called bounded derivation tree depth property (BDTDP).

Definition 9 (BDTDP). A class $\mathcal{C}$ of TGDs satisfies the bounded derivation tree depth property (BDTDP) if for each $\Sigma \in \mathcal{C}$, there exists some $k \geq 0$ such that for every $B C Q$ query $\exists \mathbf{Z} p(\mathbf{Z})$ and every database $D, D \cup \Sigma \models \exists \mathbf{Z} p(\mathbf{Z})$ iff $T(D, \Sigma) \models p(\mathbf{n})$ for some instantiated derivation tree $T(D, \Sigma)$ and atom $p(\mathbf{n})$, where $\operatorname{depth}(T(D, \Sigma)) \leq k$ and $h(\mathbf{Z})=\mathbf{n}$ for some homomorphism $h$.

Basically, Definition 9 says that if a class of TGDs satisfies BDTDP, then its every BCQ query answering problem can be always decided within a fixed number $k$ of derivation steps with respect to the corresponding instantiated derivation trees. Note that this $k$ is independent from the input database $D$ and the specific BCQ query $q$. Also note that BDTDP is different from the previous BDDP, i.e., Definition 1, which is defined based on the chase procedure.

Theorem 4. The class of LR TGDs satisfies BDTDP.

Proof. We first introduce the notion of subsumation between two derivation trees.
Definition 10 (Derivation tree subsumption). Let $\Sigma$ be a set of TGDs, and $T_{1}(\Sigma)$ and $T_{2}(\Sigma)$ be two derivation trees of $\Sigma$. Then we say that $T_{2}(\Sigma)$ subsumes $T_{1}(\Sigma)$ if the following conditions are satisfied: (1) $\operatorname{root}\left(T_{2}(\Sigma)\right)=\operatorname{root}\left(T_{1}(\Sigma)\right)$; and (2) leafNodes $\left(T_{2}(\Sigma)\right) \subset$ leafNodes $\left(T_{1}(\Sigma)\right)$.

Proof. Given a set $\Sigma$ of LR TGDs. Let $\mathcal{T}(\Sigma)$ be the set of all derivation trees of $\Sigma$. We consider the set $\mathbb{T}(\Sigma)$ of all derivation trees that are distinct under $\sim$ and their tree depths are not larger than $N$, where $N$ is the integer mentioned in Proposition 24. Then it is clear that $\mathbb{T}(\Sigma) \subseteq \mathcal{T}(\Sigma)$ and is a finite set. Now we can prove the following important result:

Lemma 2. Let $T(\Sigma) \in \mathcal{T}(\Sigma)$ (note $\Sigma$ is LR). Then for every database $D$ and every atom $p(\mathbf{t}), T(D, \Sigma) \models p(\mathbf{t})$ iff there exists some $T^{\prime}(\Sigma) \in \mathbb{T}(\Sigma)$ such that $T^{\prime}(D, \Sigma) \models p(\mathbf{t})$.

Then the theorem follows directly from Lemma 2] by setting the bound to be the maximal depth of trees in $\mathbb{T}(\Sigma)$. The key idea of proving Lemma 2 is based on the fact that for any tree $T(\Sigma)$ in $\mathcal{T}(\Sigma)$, there is a corresponding tree $T^{\prime}(\Sigma)$ in $\mathbb{T}(\Sigma)$ which can replace $T(\Sigma)$ without affecting $T(\Sigma)$ 's derivations. Without loss of generality, consider a tree $T(\Sigma)$ in $\mathcal{T}(\Sigma)$, where a path $P$ in $T(\Sigma)$ is longer than $N$. Then from Proposition 2, there must exist a loop pattern $L=\left(w_{i}, \cdots, w_{j}\right)$ in path $P$, such that the depth of node $w_{i}$ is within the bound $N$, and the depth of node $w_{j}$ is beyond $N$. Since $w_{i} \sim w_{j}$ and $L$ is loop restricted and from the conditions presented in Definition 8, then using similar ideas from (Chen et al. 2011), we can prove that the subtree underneath the node $\operatorname{bod}_{b}\left(\rho_{i}\right)$ in $T(\Sigma)$ can be replaced by the subtree underneath the node $\operatorname{bod}_{b}\left(\rho_{j}\right)$. That is, the loop pattern fragment $\left(w_{i}, \cdots, w_{j}\right)$ in path $P$ is replaced by a new node $w_{i}^{*}:\left(\alpha_{i},\left[\operatorname{bod} y_{b}\left(\rho_{j}\right), \operatorname{bod} y_{h}\left(\rho_{i}\right) \rightarrow \alpha_{i}\right]\right)$. According to Proposition 2, $\Sigma$ only has a finite number of loop patterns under $\sim$. So by doing this folding for all paths in $T(\Sigma)$, we eventually transform $T(\Sigma)$ into a $T^{\prime}(\Sigma)$ whose depth is bounded by $N$, that is, $T^{\prime}(\Sigma) \in \mathbb{T}(\Sigma)$.

The following theorem reveals an important connection between BDTDP and BDDP.

Theorem 5. If a class $\mathcal{C}$ of TGDs satisfies BDTDP then $\mathcal{C}$ also satisfies $B D D P$.

According to Theorem 5 from (Calì, Gottlob, and Pieris 2012), it is clear that the class of LR TGDs is first-order rewritable.

Theorem 6. For the class of LR TGDs, the BCQA's data complexity is in $\mathrm{AC}^{0}$, and the combined complexity is EXPTIME complete.

Theorem 7. Deciding whether a set of TGDs is loop restricted is PSPACE complete.

\footnotetext{
${ }^{4}$ A complete proof of Proposition 2 is given in the full version of this paper, in which $N$ is presented.
} 


\section{Generalisation of Loop Restricted Patterns}

As described in previous section, the notion of loop patterns provides a useful means of defining the class of LR TGDs that is first-order rewritable. Now we show that loop patterns can be employed as a unified notion to significantly extend LR TGDs to a more general class of TGDs.

Firstly, we introduce a useful notion. Let $(\alpha, \rho)$ be in a loop pattern $L$ and $B$ a set of atoms occurring in body $(\rho)$. We use notion $\operatorname{null}(B)$ to denote the set of all labelled nulls occurring in $B$.

Definition 11 (Generalised loop restricted (GLR) patterns). Let $\Sigma$ be a set of TGDs. $\Sigma$ is generalised loop restricted $(G L R)$, if each loop pattern $L=\left(\alpha_{1}, \rho_{1}\right) \cdots$ $\left(\alpha_{n}, \rho_{n}\right)$ of $\Sigma$ falls into one of the following four types:

Type I For each pair $\left(\alpha_{i}, \rho_{i}\right)$ in $L(1 \leq i<n)$, body $\left(\rho_{i}\right)$ can be separated into two disjoint parts body $\left(\rho_{i}\right)=$ body $_{\mathfrak{h}}\left(\rho_{i}\right) \cup$ body $_{\mathbf{b}}\left(\rho_{i}\right)$ such that the following three conditions holds:

1. $\operatorname{body}_{\mathbf{h}}\left(\rho_{i}\right) \cap \operatorname{body}_{\mathbf{b}}\left(\rho_{i}\right)=\emptyset$,

2. $\alpha_{i+1} \in \operatorname{body}_{\mathrm{b}}\left(\rho_{i}\right)$,

3. $\operatorname{var}\left(\left\{\alpha_{i}\right\} \cup \operatorname{body}_{\mathbf{h}}\left(\rho_{i}\right)\right) \cap \operatorname{var}\left(\operatorname{body}_{\mathbf{b}}\left(\rho_{i}\right)\right)=$ $\bigcap_{j=1}^{n} \operatorname{var}\left(\alpha_{j}\right)$;

Type II There exists a pair $\left(\alpha_{i}, \rho_{i}\right)$ in $L(1 \leq i<n)$ such that body $\left(\rho_{i}\right)$ can be separated into two disjoint parts $\operatorname{body}\left(\rho_{i}\right)=\operatorname{body}_{\mathbf{h}}\left(\rho_{i}\right) \cup \operatorname{body}_{\mathbf{b}}\left(\rho_{i}\right)$, where the following three conditions hold:

1. $\operatorname{body}_{\mathbf{h}}\left(\rho_{i}\right) \cap \operatorname{body}_{\mathbf{b}}\left(\rho_{i}\right)=\emptyset$,

2. $\alpha_{i+1} \in \operatorname{body}_{\mathbf{b}}\left(\rho_{i}\right)$,

3. $\operatorname{var}\left(\left\{\alpha_{i}\right\} \cup \operatorname{body}_{\mathbf{h}}\left(\rho_{i}\right)\right) \cap \operatorname{var}\left(\operatorname{body}_{\mathbf{b}}\left(\rho_{i}\right)\right)=\emptyset$;

Type III For each pair $\left(\alpha_{i}, \rho_{i}\right)$ in $L(1 \leq i<n)$ and each $\beta \in \operatorname{body}\left(\rho_{i}\right), \operatorname{var}\left(\rho_{i}\right) \subseteq \operatorname{var}(\beta)$;

Type IV For each pair $\left(\alpha_{i}, \rho_{i}\right)$ in $L(1 \leq i<n)$ and each $\beta$ $\in \operatorname{body}\left(\rho_{i}\right) \backslash\left\{\alpha_{i+1}\right\},\left(\operatorname{var}\left(\alpha_{i+1}\right) \cap \operatorname{var}(\beta)\right) \neq \emptyset$ implies $\left(\operatorname{var}\left(\alpha_{i+1}\right) \cap \operatorname{var}(\beta)\right) \subseteq \bigcap_{j=1}^{i} \operatorname{var}\left(\alpha_{j}\right) ;$

Type $\mathbf{V}$ There exists a pair $\left(\alpha_{i}, \rho_{i}\right)$ in $L(1 \leq i<n)$, such that body $\left(\rho_{i}\right)$ can be separated into two disjoint parts $\operatorname{body}\left(\rho_{i}\right)=\operatorname{body}_{\mathbf{h}}\left(\rho_{i}\right) \cup$ body $_{\mathbf{b}}\left(\rho_{i}\right)$, where the following three conditions hold:

1. $\operatorname{body}_{\mathbf{h}}\left(\rho_{i}\right) \cap \operatorname{body}_{\mathbf{b}}\left(\rho_{i}\right)=\emptyset$,

2. $\left(\bigcup_{j=i+1}^{n}\left(\alpha_{j}\right)\right) \cap \operatorname{body}_{\mathrm{h}}\left(\rho_{i}\right)=\emptyset$,

3. $\operatorname{null}\left(\operatorname{body}_{\mathrm{h}}\left(\rho_{i}\right)\right) \neq \emptyset$.

Let us take a closer look at Definition 11 Firstly, Type I simply specifies LR TGDs, so the class of GLR TGDs properly contains the class of LR TGDs. Type II says that for the body part of $\rho_{i}$ containing the recursive atom in the loop pattern, i.e., $\alpha_{i+1} \in$ body $_{\mathbf{b}}\left(\rho_{i}\right)$, its variables are not in common with variables occurring in the head $\alpha_{i}$ and the other part of the body body ${ }_{\mathrm{h}}\left(\rho_{i}\right)$. This indicates that recursion embedded in the underlying loop pattern will not actually happen due to the lack of shared variables.

Type III, on the other hand, says that for each rule $\rho_{i}$ in every loop pattern, all variables occurring in $\rho_{i}$ are guarded by each atom in $\rho_{i}$ 's body. Type IV concerns the shared variables occurring in both recursive and non-recursive atoms in the body of rule $\rho_{i}$ in a loop pattern, i.e., $\operatorname{var}\left(\alpha_{i+1}\right) \cap \operatorname{var}(\beta)$. It requires that all such shared variables must be passed on to all following rules in the loop pattern. Finally, Type V ensures that no cycle occurs in $\Sigma$ 's graph of rule dependencies.

Theorem 8. The class of GLR TGDs satisfies BDTDP.

According to Theorem 5, we know that the class of GLR TGDs satisfying BDTDP also satisfies BDDP, and hence the following corollary holds.

Corollary 9. The class of GLR TGDs is first-order rewritable.

Theorem 10. Consider the BCQA problem for a given set of GLR TDGs. Its data complexity is in $\mathrm{AC}^{0}$, and its combined complexity is EXPTIME complete.

Theorem 11. Deciding whether a set of TGDs is generalised loop restricted is PSPACE complete.

\section{Relationship to Other First-order Rewritable Classes}

In this section, we study the relationship between our proposed GLR TGDs class and other first-order rewritable TGDs classes. First of all, we briefly introduce these existing TGDs classes, which are known to be first-order rewritable. A TGD of the form (1):

$$
\sigma: \forall \mathbf{X Y} \varphi(\mathbf{X}, \mathbf{Y}) \rightarrow \exists \mathbf{Z} \psi(\mathbf{X}, \mathbf{Z})
$$

is called linear if $\varphi(\mathbf{X}, \mathbf{Y})$ is an atom. $\sigma$ is multi-linear if each atom in $\varphi$ contains all the universally quantified variables of $\sigma$ (Calì, Gottlob, and Lukasiewicz 2012). A set $\Sigma$ of TGDs is linear or multi-linear if each TGD in $\Sigma$ is linear or multi-linear, respectively. $\Sigma$ is acyclic if $\Sigma$ 's position graph contains no cycle (Civili and Rosati 2012), while $\Sigma$ is $a G R D$ if $\Sigma$ 's rule dependency graph contains no cycle (Baget 2004; Baget et al. 2011).

Informally, $\Sigma$ is said to have the sticky property if for each $\sigma$ in $\Sigma$, all variables occurring in $\operatorname{body}(\sigma)$ more than once also appear in head $(\sigma)$, and furthermore, also appear in every atom obtained from some chase derivation which involves head $(\sigma)$, that is, stick to all such atoms (Calì, Gottlob, and Pieris 2012). The sticky-join property, on the other hand, is less restricted than sticky property, where it only requires to stick certain variables occurring more than once in body $(\sigma)$ based on certain joinless condition. It has been showed that the sticky-join class captures both the sticky and linear classes, but is incomparable with multi-linear class (Calì, Gottlob, and Pieris 2012).

GLR actually captures a large class of first-order rewritable TGDs. Let us use LR, ML, AC, SJ, aGRD and DR to denote the classes of loop restricted, mulit-linear (Calì, Gottlob, and Lukasiewicz 2012), acyclic (Civili and Rosati 2012), sticky-join (Calì, Gottlob, and Pieris 2012), aGRD (Baget 2004; Baget et al. 2011) and domain restricted TGDs (Baget et al. 2011), respectively. Then we have the following result.

Proposition 3. Let GLR be the class of generalised loop restricted TGDs defined in Definition 11] Then we have that: (1) $\mathrm{LR} \subsetneq \mathrm{GLR}$; (2) $\mathrm{AC} \subsetneq \mathrm{GLR}$; (3) $\mathrm{ML} \subsetneq \mathrm{GLR}$; (4) $\mathrm{SJ} \subsetneq$ $\mathrm{GLR}$; (5) aGRD $\subsetneq \mathrm{GLR} ;(6) \mathrm{DR} \subsetneq \mathrm{GLR}$. 
Proof. We prove by considering the individual cases as follows:

("DR $\subsetneq$ GLR"): This follows from the fact that a TGD rule $\sigma$ is domain-restricted if each head atom $\alpha$ $\in \operatorname{Head}(\sigma)$ mentions none or all of the variables in $\operatorname{Body}(\sigma)$ (Baget et al. 2011).

("LR $\subsetneq$ GLR"): This follows from the fact that the loop pattern Type I of Definition 11 is actually the loop pattern of Definition 8 .

("AC $\subsetneq$ GLR"): On the contrary, assume that there exists some $\Sigma \in \mathrm{AC}$ such that $\Sigma \notin \mathrm{GLR}$. Then by Definition 11, there exists some loop pattern $L=\left(\alpha_{1}, \rho_{1}\right) \cdots\left(\alpha_{n}, \rho_{n}\right)$ such that it is neither of the Types I-V as described in Definition 11 In particular, we have that $L$ is not of the Type II. Then this implies that for all $\left(\alpha_{i}, \rho_{i}\right)(1<i \leq n)$, we have that body $\left(\rho_{i}\right)$ separated into two disjoint body parts body $\left(\rho_{i}\right)=\operatorname{body}_{\mathbf{h}}\left(\rho_{i}\right) \cup$ body $_{\mathbf{b}}\left(\rho_{i}\right)$ implies that for all $j(1 \leq j<i)$, one of the following conditions holds:

1. $\operatorname{body}_{\mathbf{h}}\left(\rho_{i}\right) \cap \operatorname{body}_{\mathbf{b}}\left(\rho_{i}\right) \neq \emptyset$, or

2. $\operatorname{var}\left(\left\{\alpha_{j}\right\} \cup \operatorname{body}_{\mathbf{h}}\left(\rho_{i}\right)\right) \cap \operatorname{var}\left(\operatorname{body}_{\mathbf{b}}\left(\rho_{i}\right)\right) \neq \emptyset$.

In particular, if we take body $\left(\rho_{i}\right)=\emptyset$ and body $\left(\rho_{i}\right)=$ body $\left(\rho_{i}\right)$, for each $i \in\{1, \ldots, n\}$, then since $L$ is a loop pattern (and thus, $\left.\alpha_{i+1} \in \operatorname{body}\left(\rho_{i}\right)=\operatorname{body}_{\mathbf{b}}\left(\rho_{i}\right)\right)$ then we have that Conditions 1 and 2 cannot hold. Therefore, we must have that Condition 3 holds for each $\left(\alpha_{i}, \rho_{i}\right)(1 \leq$ $i<n)$ (i.e., if we take $\operatorname{body}_{\mathrm{h}}\left(\rho_{i}\right)=\emptyset$ and $\operatorname{body}_{\mathrm{b}}\left(\rho_{i}\right)=$ body $\left.\left(\rho_{i}\right)\right)$. Then this contradicts the assumption that $\Sigma \in$ aGRD because this implies a cycle in the "firing graph" (Baget 2004).

("ML $\subsetneq$ GLR"): On the contrary, assume that there exists some $\Sigma \in \mathrm{ML}$ such that $\Sigma \notin \mathrm{GLR}$. Then again by Definition 11, there exists some loop pattern $L=$ $\left(\alpha_{1}, \rho_{1}\right) \cdots\left(\alpha_{n}, \rho_{n}\right)$ such that it is neither of the Types $\mathrm{I}-\mathrm{V}$ as described in Definition 11 In particular, we have that $L$ is not of the Type III. Then this implies that there exists some $\left(\alpha_{i}, \rho_{i}\right)(1 \leq i<n)$ such that $\operatorname{var}\left(\rho_{i}\right) \nsubseteq$ $\operatorname{var}(\beta)$, for some $\beta \in \operatorname{body}\left(\rho_{i}\right)$. Therefore, since $\rho_{i}=$ $\sigma_{i} \theta_{i}$, for some $\sigma_{i} \in \Sigma$ and assignment $\theta_{i}$, then it follows that there exists some $\beta^{\prime} \in \operatorname{body}\left(\sigma_{i}\right)$ such that $\operatorname{var}\left(\sigma_{i}\right) \not$ $\operatorname{var}\left(\beta^{\prime}\right)$. Then this contradicts the assumption that $\Sigma \in$ ML.

("SJ $\subsetneq$ GLR"): On the contrary, assume that there exists some $\Sigma \in \mathrm{SJ}$ such that $\Sigma \notin \mathrm{GLR}$. Then again by Definition 11, there exists some loop pattern $L=$ $\left(\alpha_{1}, \rho_{1}\right) \cdots\left(\alpha_{n}, \rho_{n}\right)$ such that it is neither of the Types $\mathrm{I}-\mathrm{V}$ as described in Definition [11. In particular, we have that $L$ is not of the Type IV. Then this implies that there exists some pair $\left(\alpha_{i}, \rho_{i}\right)$ in $L(1 \leq i<n)$ such that $\left(\operatorname{var}\left(\alpha_{i}\right) \cap \operatorname{var}(\beta)\right) \nsubseteq \bigcap_{j=i+1}^{n} \operatorname{var}\left(\alpha_{j}\right)$, for some $\beta \in \operatorname{body}\left(\rho_{i+1}\right) \backslash\left\{\alpha_{i}\right\}$. Then this again contradicts the assumption that $\Sigma \in$ SJ since the "expansion" of $\Sigma$ (Calì, Gottlob, and Pieris 2012) (which correspond to the loop pattern) will contain a marked variable that occurs in two different atoms;

("aGRD $\subsetneq$ GLR"): On the contrary, assume that there exists some $\Sigma \in$ aGRD and $\Sigma \notin$ GLR. Then again by Definition 11, there exists some loop pattern $L=$ $\left(\alpha_{1}, \rho_{1}\right) \cdots\left(\alpha_{n}, \rho_{n}\right)$ such that it is neither of the Types I-V as described in Definition 11 In particular, we have that $L$ is not of the Type $\mathrm{V}$. Then we have from the definition of loop restricted Type $V$ that $\Sigma$ will have cycle in the rule dependency graph, which contradicts the assumption that $\Sigma \in$ aGRD.

In (Civili and Rosati 2012), the weakly recursive (WR) class of simple TGDs was proposed. A set of TGDs $\Sigma$ is simple if for each $\sigma \in \Sigma$, each atom $\alpha$ in $\sigma$ does not have any occurrence of constants and repeated variables. For a given set $\Sigma$ of simple TGDs, Civili and Rosati considered $\Sigma$ 's position graph, and defined $\Sigma$ to be weakly recursive if $\Sigma$ 's position graph does not contain any cycles that have edges with explicit or implicit variable transitive connections. The detailed definition of WR class of simple TGDs is referred to (Civili and Rosati 2012).

It was then shown in (Civili and Rosati 2012) that the WR class captures all existing known first-order rewritable classes when restricted to simple TGDs. The following result shows that in the case of simple TGDs, WR and GLR are two incomparable first-order rewritable classes.

Proposition 4. Under the restriction to simple TGDs, we have that $\mathrm{GLR} \nsubseteq \mathrm{WR}$ and $\mathrm{WR} \nsubseteq \mathrm{GLR}$.

Proof. ("GLR $\not \subset$ WR") From the proof of Theorem 5 in (Civili and Rosati 2012), we consider a set $\Sigma$ of simple TGDs comprising of the following two rules:

$$
\begin{aligned}
& \mathrm{s}(X, Y, Z, V) \rightarrow \mathrm{r}(X, Y, Z), \\
& \mathrm{t}(X, W) \wedge \mathrm{r}(X, W, Y) \rightarrow \exists Z \mathrm{~s}(X, Y, Z, W) .
\end{aligned}
$$

Then we get that $\Sigma$ is not in the GLR class of simple TGDs.

("WR $\nsubseteq$ GLR") Consider the following set of TGDs $\Sigma^{\prime}$ :

$$
\begin{aligned}
& \mathrm{r}(X, Y) \wedge \mathrm{r}(Y, Z) \rightarrow \exists U \mathrm{~s}(X, Z, U), \\
& \mathrm{s}(X, Z, U) \wedge \mathrm{t}(X, U) \rightarrow \mathrm{t}(Z, U), \\
& \mathrm{t}(X, U) \wedge \mathrm{t}(Z, U) \rightarrow \mathrm{r}(X, Z) .
\end{aligned}
$$

Then it can be checked that $\Sigma^{\prime}$ is not WR because we will have a cycle $\langle r[], t[]\rangle,\langle t[], s[]\rangle,\langle s[], r[]\rangle$ in the "position graph" (Civili and Rosati 2012) of $\Sigma^{\prime}$ and where the edge $\langle s[], r[]\rangle$ will have both an $m$ and $s$ label. On the other hand, we have that $\Sigma^{\prime}$ is aGRD, which is also GLR by Proposition 3

We emphasize that our results presented in this paper are for arbitrary TGDs, while simple TGDs are probably restricted for representing general knowledge domains.

\section{Concluding Remarks}

Loops have been an important concept in the study for traditional Datalog programs, and then have been employed and extended in Answer Set Programming research in recent years, e.g., (Chen et al. 2011, Lin and Zhou 2004, Zhang and Zhou 2010; Zhou and Zhang 2017). In this paper, through a series of novel definitions of derivation paths, derivation trees and loop patterns, we are able to discover new decidable classes of TGDs for ontology based query 
answering using a very different idea from previous approaches.

As we have showed, the class of GLR TGDs properly contains all other first-order rewritable TGDs classes for general TGDs. We believe that our results presented in this paper will be useful in developing efficient OBDA systems for broader application domains.

\section{References}

[Baader et al. 2016] Baader, F.; Bienvenu, M.; Lutz, C.; and Wolter, F. 2016. Query and predicate emptiness in ontologybased data access. Journal of Artificial Intelligence Research 56:1-59.

[Baget et al. 2011] Baget, J.; Leclère, M.; Mugnier, M.; and Salvat, E. 2011. On rules with existential variables: Walking the decidability line. Artifificial Intelligence 175(910):1620-1654.

[Baget 2004] Baget, J. 2004. Improving the forward chaining algorithm for conceptual graphs rules. In Principles of Knowledge Representation and Reasoning: Proceedings of the Ninth International Conference (KR2004), Whistler, Canada, June 2-5, 2004, 407-414.

[Bienvenu 2016] Bienvenu, M. 2016. Ontology-mediated query answering: Harnessing knowledge to get more from data. In Proceedings of IJCAI 2016, 4058-4061.

[Calì, Gottlob, and Kifer 2008] Calì, A.; Gottlob, G.; and Kifer, M. 2008. Taming the infinite chase: Query answering under expressive relational constraints. In Proceedings of the 21st International Workshop on Description Logics (DL2008), Dresden, Germany, May 13-16, 2008.

[Calì, Gottlob, and Lukasiewicz 2012] Calì, A.; Gottlob, G.; and Lukasiewicz, T. 2012. A general datalog-based framework for tractable query answering over ontologies. J. Web Sem. 14:57-83.

[Calì, Gottlob, and Pieris 2012] Calì, A.; Gottlob, G.; and Pieris, A. 2012. Towards more expressive ontology languages: The query answering problem. Artif. Intell. 193:87128.

[Chen et al. 2011] Chen, Y.; Lin, F.; Zhang, Y.; and Zhou, Y. 2011. Loop-separable programs and their first-order definability. Artificial Intelligence 175(3-4):890-913.

[Civili and Rosati 2012] Civili, C., and Rosati, R. 2012. A broad class of first-order rewritable tuple-generating dependencies. In Proceedings of the 2nd International Conference on Datalog in Academia and Industry (Datalog-2012), 6880.

[Deutsch, Nash, and Remmel 2008] Deutsch, A.; Nash, A.; and Remmel, J. B. 2008. The chase revisited. In Proceedings of the Twenty-Seventh ACM SIGMOD-SIGACTSIGART Symposium on Principles of Database Systems, PODS 2008, June 9-11, 2008, Vancouver, BC, Canada, 149158.

[Eiter, Lukasiewicz, and Predoiu 2016] Eiter, T.; Lukasiewicz, T.; and Predoiu, L. 2016. Generalized consistent query answering under existential rules. In Proceedings of KR 2016, 359-368.
[Fagin et al. 2005] Fagin, R.; Kolaitis, P. G.; Miller, R. J.; and Popa, L. 2005. Data exchange: semantics and query answering. Theor. Comput. Sci. 336(1):89-124.

[Gottlob, Manna, and Pieris 2013] Gottlob, G.; Manna, M.; and Pieris, A. 2013. Combining decidability paradigms for existential rules. Theory and Practice of Logic Programming 16(1):877-892.

[Grau et al. 2013] Grau, B. C.; Horrocks, I.; Krotzsch, M.; Kupke, C.; Magka, D.; Motik, B.; and Wang, Z. 2013. acyclicity notions for existential rules and their application to rqquery raanswering in ontologies. Journal of Artificial Intelligence Research 47:741-808.

[Hansen et al. 2015] Hansen, P.; Lutz, C.; Seylan, I. .; and Wolter, F. 2015. Efficient query rewriting in the description logic el and beyond. In Proceedings of IJCAI 2015, 30343040 .

[Kaminski, Nenov, and Grau 2014] Kaminski, M.; Nenov, Y.; and Grau, B. C. 2014. Computing datalog rewritings for disjunctive datalog programs and description logic ontologies. In Web Reasoning 2014, 76-91.

[Kontchakov, Rodriguez-Muro, and Zakharyaschev 2013] Kontchakov, R.; Rodriguez-Muro, M.; and Zakharyaschev, M. 2013. Ontology-based data access with databases: A short course. In Reasoning Web 2013, 194229.

[Krötzsch and Rudolph 2011] Krötzsch, M., and Rudolph, S. 2011. Extending decidable existential rules by joining acyclicity and guardedness. In Proceedings of IJCAI 2011, 963-968.

[Leone et al. 2012] Leone, N.; Manna, M.; Terracina, G.; and Veltri, P. 2012. Efficiently computable datalog $\exists$ programs. In Principles of Knowledge Representation and Reasoning: Proceedings of the Thirteenth International Conference, KR 2012, Rome, Italy, June 10-14, 2012.

[Lin and Zhou 2004] Lin, F., and Zhou, Y. 2004. Assat: Computing answer sets of a logic program by sat solvers. Artificial Intelligence 157:115-137.

[Nikolaou et al. 2017] Nikolaou, C.; Kostylev, E. V.; Konstantinidis, G.; Kaminski, M.; Grau, B. C.; and Horrocks, I. 2017. The bag semantics of ontology-based data access. In https://arxiv.org/abs/1705.07105.

[Patel-Schneider and Horrocks 2007] Patel-Schneider, P. F., and Horrocks, I. 2007. A comparison of two modelling paradigms in the semantic web. Journal of Web Semantics 5(4):240-250.

[Zhang and Zhou 2010] Zhang, Y., and Zhou, Y. 2010. On the progression semantics and boundedness of answer set programs. In Proceedings of the 12th International Conference on the Principles of Knowledge Representation and Reasoning (KR-2010), 518-527.

[Zhou and Zhang 2017] Zhou, Y., and Zhang, Y. 2017. A pregression semantics for first-order logic programs. Artificial Intelligence to appear. 